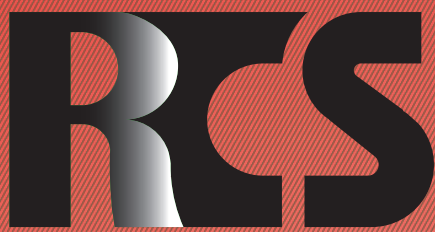

Depósito legal ppi $201502 Z U 4662$

Esta publicación científica en formato digital es continuidad de la revista impresa Depósito Legal: pp $197402 Z$ Z789

- ISSN: 1315-9518 • ISSN-E: 2477-9431

Revista de Ciencias Sociales

Universidad del Zulia. Revista de la Facultad de Ciencias Económicas y Sociales Vol. XXVII,

No. 4,2021

Esta publicación científica en formato digital es continuidad de la revista impresa Depósito Legal: pp $197402 Z 1789$ ISSN: $1315-9518$ 


\title{
Cadena de valor de la pinchagua y su productividad: Sitio Los Arenales parroquia Crucita-Ecuador
}

\author{
Zambrano Montesdeoca, Jasson Luis* \\ Palacios Cedeño, Nohemí Monserrate** \\ Ceme Macías, Cristina Auxiliadora ${ }^{* * *}$

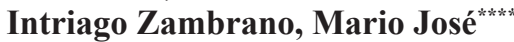

\section{Resumen}

El presente trabajo investigativo se desarrolla en el Sitio Los Arenales de la parroquia Crucita, del cantón Portoviejo, provincia de Manabí, Ecuador, tuvo como objetivo analizar la cadena de valor de la pinchagua y su productividad en el sitio Los Arenales. Se realizó un estudio a través de metodologías descriptivas y de campo. En relación a los tipos de investigación se encontraron presentes el cuantitativo y cualitativo, en donde, se aplicó la fórmula muestral, a los representantes de las organizaciones, cooperativas, asociaciones, habitantes del sector. Los resultados evidencian que la mayoría de las personas que se dedican a estas actividades se encuentran asociadas, sin embargo, pocos son los beneficios que se reciben por la propia gestión de dichas asociaciones, donde las capacitaciones, créditos y seguros han sido por gestión de las Instituciones del ramo como la Subsecretaría de Recursos Pesqueros. En conclusión, el nivel de productividad por persona es considerablemente aceptable, el cual podría variar dependiendo del nivel de obtención del producto, unidades producidas e insumos empleado. Respecto al nivel de productividad con relación al salario mensual es considerablemente alto, debido que en el barco una persona produce en dólares la cantidad de 5,32 tinas.

Palabras clave: Cadena de valor; productividad; pinchagua; indicadores; Los Arenales-Ecuador.

\footnotetext{
* Master en Dirección y Asesoramiento Financiero. Economista. Profesor Investigador de la Universidad Técnica de Manabí, Ecuador. E- mail: jasson.zambrano@utm.edu.ec (iD) ORCID: https://orcid. org/0000-00020501-8918

** $\quad$ Master en Dirección y Asesoramiento Financiero. Economista. Profesor Investigador de la Universidad Técnica de Manabí, Ecuador. E- mail nohemi.palacios@utm.edu.ec (iD ORCID: https://orcid.org/00000002-0666-5164

**** Ingeniera Comercial. Universidad Técnica de Manabí, Ecuador. E-mail: cristina.ceme93@gmail.com (iD) ORCID: https://orcid.org/0000-0003-4792-4465

***** Ingeniera Comercial. Universidad Técnica de Manabí, Ecuador. E-mail: intriago mario@gmail.com (iD) ORCID: https://orcid.org/0000-0002-4683-6378
}

Recibido: 2021-06-03 - Aceptado: 2021-08-21 


\title{
Pinchagua's value chain and its productivity at the los arenales site of the crucita parish
}

\begin{abstract}
The present investigative work is developed in the Los Arenales Site of the Crucita parish, of the Portoviejo canton, Manabí province, Ecuador, its objective was to analyze the value chain of the pinchagua and its productivity in the Los Arenales site. A study was carried out through descriptive and field methodologies. Regarding the types of research, the quantitative and qualitative were present, where the sample formula was applied to representatives of organizations, cooperatives, associations, inhabitants of the sector. The results show that most of the people who dedicate themselves to these activities are associated, however, few are the benefits that are received by the management of these associations, where the training, credits and insurance have been by management of the Institutions of the branch such as the Undersecretariat of Fishing Resources. In conclusion, the level of productivity per person is considerably acceptable, which could vary depending on the level of obtaining the product, units produced and inputs used. Regarding the level of productivity in relation to the monthly salary, it is considerably high, because on the ship a person produces 5.32 tubs in dollars.
\end{abstract}

Keywords: Value chain; productivity; pinchagua; indicators; Los Arenales-Ecuador.

\section{Introducción}

La pinchagua es un tipo de pez que pertenece a la familia del arenque. Según González et al. (2017), las pinchaguas "forman parte de los recursos pesqueros de mayor importancia económica y social del Ecuador, y su actividad extractiva, desembarques, procesamiento" (p.1) como son los enlatados y harina de pescado, así como exportaciones, genera un rubro importante de divisas para el país.

Según Pazmiño-Morteira y VeraSantana (2020), mencionan que en el sitio Los Arenales se ha constituido en una de las principales caletas pesqueras que abastece de materia prima a la industria de conservas de sardina, quienes destinaban esta especie marina a grandes industrias de Manta y Guayas, y a su vez la envasaban y vendían el producto. Así mismo, dentro de la cooperativa de producción pesquera artesanal Los Arenales, en una sola embarcación se produce la cantidad de 45 toneladas de pinchagua mensuales, lo que equivale a una producción de $\$ 36.000$ dólares mensuales.

Por su parte, en el Banco Central del Ecuador (BCE, 2019), se evidencia que entre el periodo 2014 al 2018 existe una pendiente positiva, dado que en 5 años el saldo por exportaciones paso de $\$ 2.822$ miles a $\$ 3.563$ miles, lo que representa un crecimiento del $26,25 \%$; se enfatiza que las exportaciones de productos pesqueros en el año 2018 ascendieron a US\$ 1.601 millones, incluyendo productos de pinchagua, crustáceos, atún, y sardinas, un valor casi US\$ 82 millones por encima de lo exportado en el 2017. En 2018, Ecuador exportó la mayor parte de sus productos relacionados con la pesca a Vietnam $25 \%$, seguido de Estados Unidos con 18\%. Los principales países a los cuales Ecuador exporta son: Vietnam, Estados Unidos, China, España, Francia e Italia.

En Ecuador existen 200 caletas pesqueras, dentro de éstas 43 , se encuentran entre los $350 \mathrm{~km}$ de playa que hay en Manabí, desde Pedernales hasta Puerto López. La tonelada de la pinchagua limpia, libre de escamas, sin cabeza y vísceras se vende a 
USD 600 a los comerciantes, quienes venden a las 14 procesadoras en Manta, Montecristi y Jaramijó; allí se elaboran las sardinas en conservas. Los desperdicios de la sardina se acumulan en grandes camiones y se transportan a las tres fábricas de harina de pescado de Manta y de Jaramijó.

La distribución de pinchagua, está comprendida desde el norte de Bahía de Caráquez en la provincia de Manabí, Guayas y Esmeralda. En el sitio "Los Arenales", de la Parroquia Crucita, la pesca tradicional es la más difundida, aunque últimamente se ha incrementado la pesca de subsistencia como consecuencia del desempleo y la crisis económica.

Según González y Solís (2010), el sector pesquero del sitio Los Arenales de la parroquia Crucita es el segundo más productivo de Manabí y en el país, la actividad extractiva de la pinchagua en dichos sitios genera aproximadamente cinco mil puestos de trabajo directo e indirectamente relacionadas con esta actividad.

Respecto a la justificación de la presente investigación, resulta ser un gran aporte para la sociedad en general, más específicamente al sector pesquero, puesto que contribuye en gran medida en lo social y productivo. Desde el punto de vista social, se conoció las condiciones laborales que presentan los pescadores del sector Los Arenales; y productivo, porque se aportó con información base para instruir en mejores procesos de ordenamiento de dicha actividad que involucren a los pescadores. En ese sentido, el objetivo de este estudio consistió en analizar la cadena de valor de la pinchagua y su relación con la productividad en el sitio Los Arenales de la parroquia Crucita del cantón Portoviejo, Ecuador. Al respecto, sostienen Álvarez et al. (2020) que:

Ecuador cuenta con recursos estratégicos para facilitar la producción y comercialización de bienes y servicios y de esta forma, incrementar la economía del mercado nacional. A la vez, trabaja en potenciar su desarrollo, a partir de una economía activa, en la que se propicien actividades productivas sustentables en beneficio de las comunidades que forman parte de los diferentes cantones y provincias del país. (p.354)

\section{Cadena de Valor}

De acuerdo con Donovan (2006), la cadena de valor "representa la articulación de todos los actores involucrados en la producción, transformación y comercialización de un producto, desde la producción primaria, pasando por diferentes niveles de transformación e intermediación, hasta el consumo final, acompañado por los proveedores de servicios" (p.2), técnicos, empresariales, así como financieros de dicha cadena.

La idea de una cadena de valor, está centrada en las actividades necesarias para convertir la materia prima en productos terminados y venderlos (Organización de las Naciones Unidas para la Alimentación y la Agricultura [FAO], 2015), y en el valor que se agrega en cada eslabón. Sin embargo, a pesar de los múltiples conceptos, se considera a Michael E. Porter como el padre de la cadena de valor, por ser el primero en hacer planteamientos teóricos congruentes y novedosos en torno a este concepto. Para Porter (2006), la cadena de valor es una herramienta o medio sistemático que permite analizar las fuentes de la ventaja competitiva, es decir, la cadena de valor permite dividir a la empresa en sus actividades estratégicamente relevantes a fin de comprender su comportamiento en costos,

\subsection{Cadena de valor de la Pinchagua}

La cadena de valor permite a la cooperativa y a la asociación, conocer sus productos desde la obtención hasta el consumo, lo que facilita la creación de alianzas tanto productivas como de distribución y mercadeo, ayudando a identificar, así como solucionar problemas que surgen a lo largo del proceso productivo, tal como se ilustra en la Figura I. 


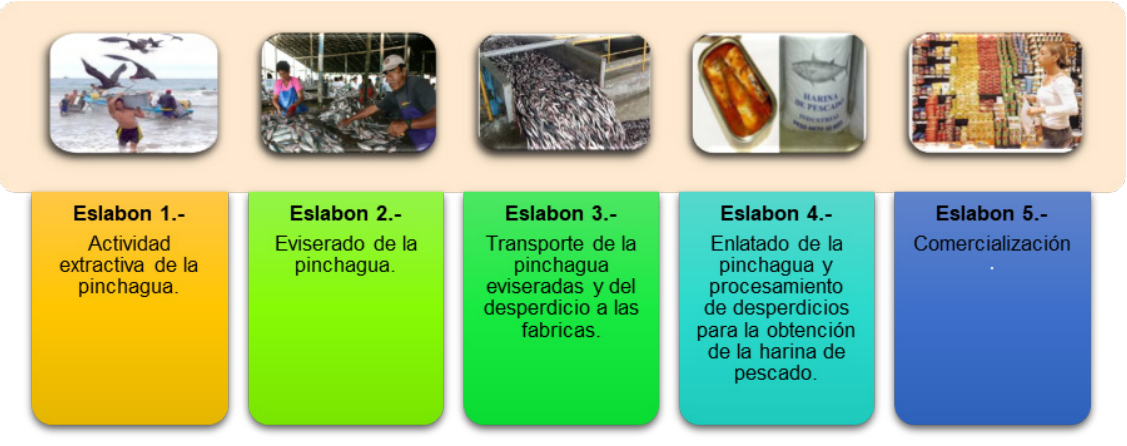

Fuente: Elaboración propia, 2020.

Figura I: Cadena de valor de la pinchagua

En la Figura I, se pueden apreciar en forma resumida los 5 eslabones en los que se divide la cadena de valor de la pinchagua, los cuales se detallan a continuación:

\section{a. Eslabón 1: Actividad pesquera extractiva de la pinchagua}

En la Figura II, se visualiza la extracción que se realiza mediante un aparato tecnológico llamado "sonar" que aprovecha la propagación del sonido bajo el agua, que funciona como un radar en la superficie acuática. De acuerdo con Rodríguez (2017), son manchas que determinan a los peces correr, y de esta manera poder capturarlos, por lo general, esta jornada se realiza en la noche o en la madrugada, ya sea, en barcos o embarcaciones más pequeñas.

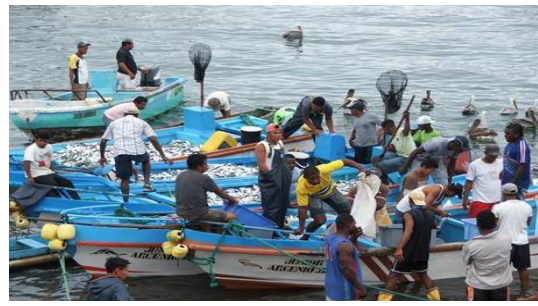

Fuente: Elaboración propia, 2020.

Figura II: Extracción de la pinchagua

\section{b. Eslabón 2: Eviscerado de la pinchagua}

El segundo eslabón, corresponde al eviscerado de la pinchagua, el cual consiste en quitar las escamas, las vísceras y las cabezas a la pinchagua, en esta actividad se emplean principalmente mujeres de la zona, las que cobran por tinas o baldes de pinchagua limpia, actividad que se realiza, en tarimas o ramadas, tal como se muestra en la Figura III.

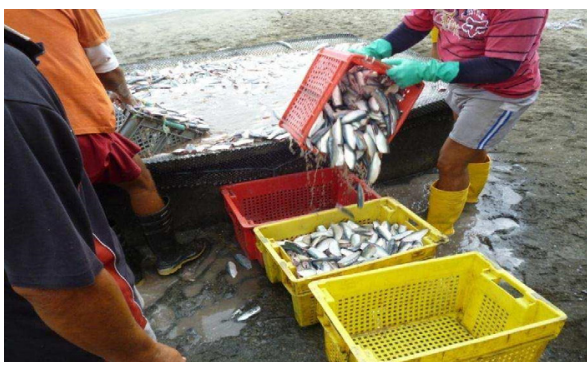

Fuente: Elaboración propia, 2020.

Figura III: Eviscerado de la pinchagua 


\section{c. Eslabón 3: Transporte de las pinchaguas evisceradas y del desperdicio a las fabricas}

Como tercer eslabón y como se evidencia en las Figuras IV y V corresponde al transporte de la pinchagua, esta se realiza en dos actividades diferentes; la que esta evisceradas se lleva a la fábrica para ser sardina en lata; y la otra, que es desperdicio se lleva a la fábrica para convertirla en harina.

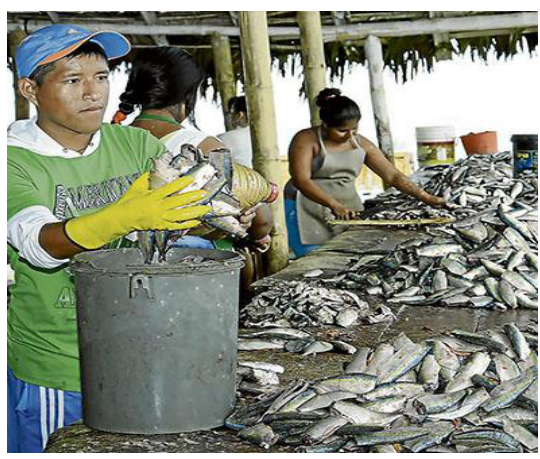

Fuente: Elaboración propia, 2020.

Figura IV: Transporte de la pinchagua

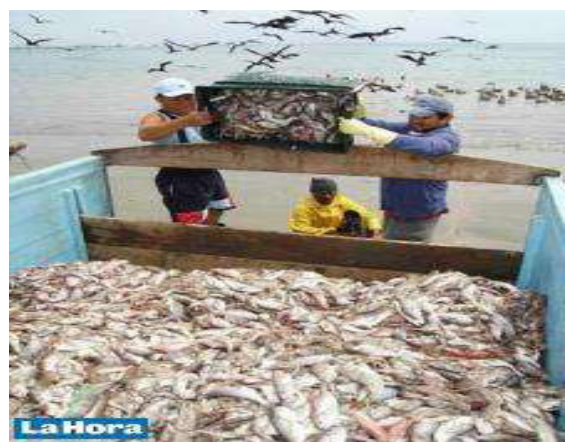

Fuente: Elaboración propia, 2020.

Figura V: Separacion de la pinchagua y del desperdicio

\section{d. Eslabón 4.- Enlatado de la pinchagua y procesamiento de desperdicios para la obtención de la harina de pescado}

En la Figura VI, se identifica el enlatado de la pinchagua y procesamiento de desperdicios para la obtención de la harina de pescado. Como cuarto eslabón, se encuentra el enlatado de la pinchagua en salsa de tomate o alguna clase de aceite, este proceso lo realizan las fábricas, destinadas a tal actividad.

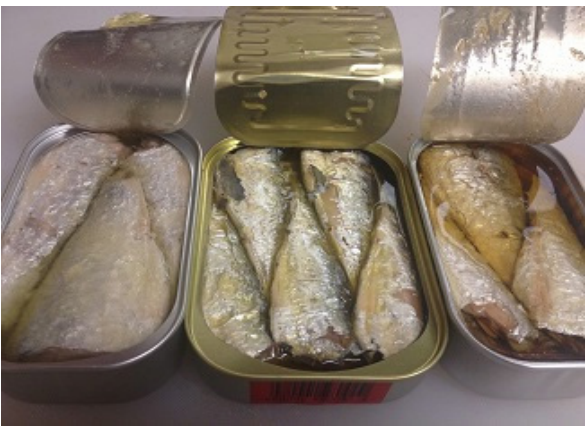

Fuente: Elaboración propia, 2020. Figura VI: Enlatado de la pinchagua

\section{e. Eslabón 5: Comercialización}

Como quinto eslabón, tal como se evidencia en la Figura VII, corresponde a la comercialización, en el cual, van destinadas a las empresas procesadoras a las que llega el producto, y estas son principalmente la empresa Real, Inepaca, Isabel, Lubar; a estas empresas se destinan el producto convertido en materia prima, puesto que los desperdicios van destinados a la fábrica Tadel, misma que se dedica al procesamiento de harina y aceite de pescado. 


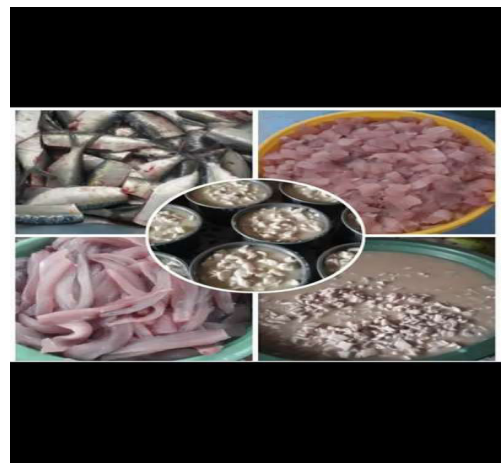

Fuente: Elaboración propia, 2020.

\section{Figura VII: Comercialización de la pinchagua}

\section{Productividad}

Respecto a la productividad, García (2011) menciona que la misma, es la relación entre los productos logrados y los insumos que se utilizaron o los factores de la producción que intervinieron, es decir, la relación entre la actividad productiva y los medios que son necesarios para conseguirlo. De acuerdo con Fontalvo, De La Hoz y Morelos (2018), "cuando se analiza la proporción de los recursos utilizados y se compara con los resultados obtenidos, se habla de productividad; un término relacionado también con los términos de eficiencia y eficacia" (p.49).

Por su parte Villegas et al. (2020), señala que se vincula directamente la "eficiencia o la productividad como principal elemento explicativo del crecimiento económico y de las diferencias entre países y economías" (p.328329), siendo estos medios del tipo tecnológicos, humanos o de infraestructuras. También puede ser entendida como la producción obtenida por un sistema productivo usando determinados recursos para este fin.

Así mismo, se puede argumentar que la productividad es un concepto genérico, que se adapta fácilmente a cualquier campo de la vida social, es un término que describe la destreza que tiene el componente de una organización que genera una ganancia o un producto efectivo. Por su parte, ZambranoAlcívar y Zambrano-Castro (2020), definen como productividad la relación que hay entre lo que se produce y los medios utilizados para conseguirlo, tal como la mano de obra, el capital, los materiales o el tiempo.

Los indicadores de productividad, según Galarza y Kámiche (2015) agregan que los mismos se relacionan con la medida exacta de la eficacia y eficiencia de los procesos en las empresas. De acuerdo a Monroy y Simbaqueaba (2017), representan la herramienta más adecuada para ejercer el control dentro de una organización. En ese sentido, según Miranda y Toirac (2010), la clasificación de los indicadores de productividad, se presenta de la siguiente manera:

a. Productividad total: Unidades producidas/ insumo empleado

b. Productividad en función del salario: Unidades producidas/ salario mensual

c. Productividad laboral: Unidades producidas / horas - hombres empleados

d. Productividad del capital: Salidas / mano de obra + material +energía + capital + otros.

Es importante recalcar que mientras mayor sea el índice de productividad, mejor será el logro de las metas, teniendo en claro diversos puntos a favor acerca de la productividad, tales como: Producir la misma cantidad con menos hombres, producir más cantidad con menos hombres, o producir más cantidad con la misma cantidad de hombres.

\section{Metodología}

En esta investigación se utilizó los siguientes métodos: El deductivo, para obtener las debidas conclusiones generales a este estudio; así mismo, el método analítico, para segmentar de un todo, e ir descomponiendo en partes o elementos, en la que se observó las causas, la naturaleza y hasta los efectos de esta investigación.

En cuanto a los tipos de investigación se contempla el cualitativo y cuantitativo, siendo 
necesario recurrir a información numérica o base de datos, y análisis de información teórica del entorno en el que se desarrolla el objeto de estudio, se hizo registros narrativos acerca de los fenómenos que se estudiaron mediante técnicas investigativas, como la observación, entrevista y encuestas, a fin de realizar las tablas y representaciones estadísticas. $\mathrm{La}$ población o universo para esta investigación, corresponde a los pescadores artesanales del sitio Los Arenales, el cual consta de 4.100 pescadores, del que se extrajo una pequeña porción, el cual corresponde a 96 pescadores, que surgió del cálculo de la muestra, a partir de la siguiente ecuación:

$$
n=\frac{N}{E^{2}(N-1)+1}
$$

En donde: $\mathrm{n}=$ muestra; $\mathrm{N}=$ población; $\mathrm{E}=$ Error muestral, que será del $10 \%=0,10 ; \mathrm{y}$ el resultado obtenido es el siguiente:

$n=\frac{4100}{(0,10)^{2}(4100-1)+1}=\frac{4100}{0,01(4099)+1}=\frac{4100}{41,99}$

$$
=95,50
$$

Pescadores.

Se aplicó la encuesta a los pescadores que laboran dentro del sector Los Arenales; luego, se procesó la información en la aplicación de Excel, la cual se presenta en tablas de frecuencias estadísticas; posteriormente, se realizó una entrevista a 5 autoridades, en las que constan el gerente de la cooperativa como también, asociaciones, y dueños de barcos propios del sector, permitiendo la validación, uso adecuado de los materiales, así como métodos utilizados en este estudio.

\section{Resultados y discusión}

Alrededor del negocio se forma un engranaje económico que deja ganancia a todos, desde el dueño de barco que es el armador, hasta quienes se encargan de filetear el pescado; así también, el barco va en busca de la pesca, de la pinchagua y de otros peces pelágicos en la zona costera de la provincia de Manabí y Santa Elena, allí trabajan entre 13 a 15 tripulantes en el barco, incluido el capitán; 3 lanchas (pangas) con 2 tripulantes cada una, quienes ganarán 348 dólares (entre todos) por cada tonelada pescada, en un tiempo de labor de 12 a 13 horas. El dueño del barco vende cada tonelada de pinchagua con cabeza en 840 dólares. Luego regresan cerca de la orilla, hasta donde se acercan las lanchas de fibra de vidrio, cada una transporta una tonelada (70 tinas) hasta la tierra.

Normalmente al momento de la evisceración de la pinchagua, se necesita un minino de 30 personas, esto lo realiza en las tarimas, las personas que trabajan suman alrededor de 4,50 dólares, en un tiempo de 1 hora o 1 hora y media. El producto limpio es llevado a las fábricas de Manta, Montecristi o Guayaquil. A continuación, se presentan las tablas, correspondiente a la encuesta realizada a los pescadores de este sector, mostrando los siguientes resultados.

\subsection{Resultados de encuesta a los pescadores artesanales del sector Los Arenales de Crucita, Ecuador}

En la Tabla 1, se muestra que alrededor del $44 \%$ del total de los encuestados, indican que el destino de sus productos son los intermediarios, puesto que así se evitan tramites que retrasan o impiden el adecuado proceso de su producto como es el caso de la pinchagua. Existe una pequeña proporción que representa el $6 \%$ del total de los encuestados, que manifiesta que son otros los destinos, aquellos que no fueron proporcionados por ser confidencial por parte de ellos. 
Tabla 1

Destino de su producto

\begin{tabular}{lll}
\hline Opción & Frecuencia & Porcentaje \\
\hline Industria & 31 & $32 \%$ \\
Intermediarios & 42 & $44 \%$ \\
Supermercados & 17 & $18 \%$ \\
Otros & 6 & $6 \%$ \\
Total & $\mathbf{9 6}$ & $\mathbf{1 0 0 \%}$ \\
\hline
\end{tabular}

Fuente: Elaboración propia, 2020.

De igual manera, en la Tabla 2 se aprecia que alrededor del $63 \%$, indica que su relación con los proveedores, intermediarios y consumidores, es buena, de hecho, tanto el pescador como el cliente valoran una buena atención, por lo que generan un efecto bastante efectivo.

Tabla 2

\section{La relación con lo demás actores de la cadena de valor}

\begin{tabular}{lcc}
\hline Opción & Frecuencia & Porcentaje \\
\hline Malo & 10 & $10 \%$ \\
Regular & 24 & $25 \%$ \\
Bueno & 60 & $63 \%$ \\
Excelente & 2 & $2 \%$ \\
Total & 96 & $100 \%$ \\
\hline
\end{tabular}

Fuente: Elaboración propia, 2020.

De acuerdo a los datos que se indican en la Tabla 3, ninguno de los pescadores manifestó que sus ingresos por esta actividad sean altos. Así mismo, alrededor del 51\% de los pescadores, declaran que los ingresos generados por esta actividad productiva son medios, esto debido en gran parte a la existencia de poca obtención de la pinchagua, en la zona costeras de Manabí o fuera de ello, lo que hace que sus ingresos no sean los esperados.

\section{Tabla 3}

Los ingresos generados por su actividad pesquera

\begin{tabular}{lll}
\hline Opción & Frecuencia & Porcentaje \\
\hline Altos & 0 & $0 \%$ \\
Medio & 49 & $51 \%$ \\
Bajos & 47 & $49 \%$ \\
Total & $\mathbf{9 6}$ & $\mathbf{1 0 0} \%$ \\
\hline
\end{tabular}

Fuente: Elaboración propia, 2020.

Asimismo, según los datos presentados en la Tabla 4, por un lado, el $2 \%$ de los encuestados, manifiestan que el tipo de problema que se presenta en su actividad es falta de papeles en reglas o por problemas de su embarcación. Por otro lado, el 49\% del total de los entrevistados, manifiestan que el tipo de problema que se observan en esta actividad es otros medios, entre los que se mencionan la contaminación, la sobreexplotación, la pesca con bomba, entre otros, que de alguna manera representan un problema para la actividad pesquera.

\section{Tabla 4}

\section{El tipo de problemas presenta en su actividad productiva}

\begin{tabular}{lll}
\hline Opción & Frecuencia & Porcentaje \\
\hline $\begin{array}{l}\text { Problema en la } \\
\text { actividad }\end{array}$ & 2 & $2 \%$ \\
$\begin{array}{l}\text { Dificultad en el } \\
\text { transporte }\end{array}$ & 39 & $41 \%$ \\
$\begin{array}{l}\text { Desventaja en la } \\
\text { comercialización }\end{array}$ & 8 & $8 \%$ \\
$\begin{array}{l}\text { Otros } \\
\text { Total }\end{array}$ & 47 & $49 \%$ \\
\hline
\end{tabular}

Fuente: Elaboración propia, 2020.

Mediante la Tabla 5, se visualiza que 
alrededor del $79 \%$ del total de los encuestados, manifiestan que los factores que afectan la comercialización de esta actividad es la inestabilidad de los precios, puesto que según ellos provoca que el pescado no se venda, perjudicándolos a largo plazo a los habitantes más pobres y vulnerables de la ciudad. Por otra parte, se encuentra el $8 \%$ del total de los encuestados, el cual, indican que los factores que afectan la comercialización de esta actividad es la sobreproducción, es claro que en cierta época del año ocurre este fenómeno, lo que provoca un exceso de oferta, conllevando a que los precios del producto sean bajos, y de alguna manera no se obtenga lo debido.

Tabla 5

\section{Factores que se consideran afecta a la comercialización de actividad}

\begin{tabular}{lll}
\hline Opción & Frecuencia & Porcentaje \\
\hline $\begin{array}{l}\text { Inestabilidad de los } \\
\text { precios }\end{array}$ & 76 & $79 \%$ \\
$\begin{array}{l}\text { Sobreproducción } \\
\begin{array}{l}\text { Difícil acceso a } \\
\text { mercados }\end{array}\end{array}$ & 8 & $8 \%$ \\
Total & 12 & $13 \%$ \\
\hline
\end{tabular}

Fuente: Elaboración propia, 2020.

\subsection{Resultados de entrevista a las autoridades y dueño de embarcaciones pesqueras}

A continuación, se presenta los resultados de la entrevista realizada a las autoridades y dueños de la embarcación pesquera:

\section{1. ¿Cuál es el principal problema que tiene en la comercialización de la pinchagua?}

Es al momento de trasladarlo a la fábrica, el inconveniente es que ya muchas de ellas han cerrado y en las pocas que hay están abastecidas o se tiene que esperar para poder entregar su pescado. Además, se tiene que esperar días o meses para el pago de la entrega de la pinchagua.

\section{2. ¿Cuánto se pesca?}

La pesca es incierta en estos tiempos, pero para dar un promedio por oscura en relación a la pinchagua se puede decir unas 700 tinas.

\section{3. ¿Cuánto entra en la producción?}

Esta producción se divide en dos partes, la primera, es la evisceración de la pinchagua que se la lleva a la fábrica para la sardina en lata; y la otra parte, es el desperdicio que se transporta a la fábrica para realizar harina de pescado.

\section{4. ¿Cuánto sale en la producción?}

Si se hace referencia a las 700 tinas de pinchagua, se puede mencionar que ya eviscerada sale el $55 \%$, es decir, 385 tinas para la fábrica, en relación al desperdicio sale el $35 \%$, que corresponde 245 tinas para la harina de pescado, y faltaría un $10 \%$ esto se debe a la sangre y otros derivados.

\subsection{Indicadores de productividad}

a. Productividad total: Unidades producidas/ insumo empleado.

Se conoce que las unidades producidas en este caso son de alrededor de 700 tinas que son equivalente a 1 barco, cantidad que puede variar. Es necesaria la utilización de aproximadamente 64 personas, correspondiente a insumos empleados, los cuales se dedican a los diferentes roles de trabajo en esta actividad productiva de la pinchagua, tal como se aprecia en la Tabla 6. 


\section{Tabla 6}

Productividad Total

\begin{tabular}{llll}
\hline $\begin{array}{l}\text { N }{ }^{\circ} \text { DE } \\
\text { TRABAJADORES }\end{array}$ & CARGOS & $\begin{array}{l}\text { Producción } \\
\text { Mensual } \\
\text { (Tinas) }\end{array}$ & Total \\
\hline 15 & Barco & 700 & 46,67 \\
6 & Pangas & 700 & 116,67 \\
5 & Cargadores & 700 & 140,00 \\
30 & Evisceradores & 700 & 23,33 \\
RECOLECTORES & Tinas de pinchagua & 385 & 192,50 \\
2 & Tinas de desperdicios & 245 & 122,50 \\
2 & & & 192,50 \\
CHOFER & Tinas de pinchagua & 385 & 122,50 \\
2 & Tinas de desperdicios & 245 & \\
\hline 64 & & & \\
\hline
\end{tabular}

Fuente: Elaboración propia, 2020.

De acuerdo a la aplicación de la fórmula, en este indicador se puede identificar que el nivel de productividad por persona en el barco es de 47 tinas, promedio que en relación a la cantidad de personas empleadas como es en este caso 15 pescadores, es considerablemente aceptable, lo cual, podría variar dependiendo del nivel de obtención del producto, y con ellos tanto las unidades producidas como el insumo empleado.

b. Productividad en función del salario: Unidades producidas/salario mensual.

A continuación, se presenta el desarrollo de varias tablas, en donde se visualiza el nivel de productividad, con respecto a los barcos, pangueros, cargadores, eviseradores, recolectores, choferes, y otros gastos, aquellas que representan un nivel variado de la misma. De acuerdo a la productividad en función al salario, se consideran los valores de las unidades producidas con el salario mensual, obteniendo como resultado que el pago de cada trabajador, excluido el capitán, es de $\$$ 403,20 por cada extracción de la pinchagua, que son equivalente a 700 tinas de pinchagua, como se visualiza en la Tabla 7.

\section{Tabla 7}

Productividad en función del salario con respecto a los barcos

\begin{tabular}{ll}
\hline Indicadores & Resultado \\
\hline Descripción & Barco \\
No. de Trabajadores & 15 \\
Producción Mensual (Tinas) & 700 \\
Valor & 15,00 \\
Total & $\$ 10.500$ \\
\% de Descuento (22\%) & $\$ 2.310,00$ \\
Pago a tripulación & $\mathbf{\$ 8 . 1 9 0 , 0 0}$ \\
\% de descuento (18\%) & $\$ 1.474,20$ \\
Pago total a capitanes & $\mathbf{\$ 2 . 9 4 8 , 4 0}$ \\
Total a pagar a trabajadores & $\mathbf{5 . 2 4 1 , 6 0}$ \\
Pago a C/Trabajador & $\$ 403,20$ \\
(Excluido el capitán) & $\mathbf{1 , 7 4}$ \\
Total de Productividad & \\
\hline
\end{tabular}

Fuente: Elaboración propia, 2020.

Con respecto a la productividad en función del salario de los pangueros y cargadores, el pago por tina es de $\$ 1,00$ por una producción de 700 tinas, correspondiente al pago a cada trabajador de la panga de $\$$ 116,67; no obstante, en los cargadores el pago por tina es de $\$ 0,75 \mathrm{ctvs}$, por una producción de la misma cantidad de tinas, correspondiendo al pago a cada trabajador en el proceso de carga de la pinchagua de $\$ 105,00$, el cual, se visualiza en la Tabla 8. 


\section{Tabla 8}

\section{Productividad en función del salario de los pangueros y cargadores}

\begin{tabular}{ccccccc} 
Descripción & $\begin{array}{c}\mathbf{N}^{\circ} \text { de } \\
\text { Trabajadores }\end{array}$ & $\begin{array}{c}\text { Producción } \\
\text { Mensual } \\
\text { (Tinas) }\end{array}$ & $\begin{array}{c}\text { Pago Por } \\
\text { tina }\end{array}$ & Valor & $\begin{array}{c}\text { Pago a c/ } \\
\text { Trabajador }\end{array}$ & $\begin{array}{c}\text { Total de } \\
\text { Productividad }\end{array}$ \\
\hline Pangueros & 6 & 700 & $\$ 1,00$ & $\$ 700,00$ & $\$ 116,67$ & $\mathbf{6 , 0 0}$ \\
\hline Cargadores & 5 & 700 & $\$ 0,75$ & $\$ 525,00$ & $\$ 105,00$ & $\mathbf{7 , 0 0}$ \\
\hline
\end{tabular}

Fuente: Elaboración propia, 2020.

En la productividad en función del salario para eviscerado y recolectores, el pago a cada trabajador, va a depender del número de tinas que recolecten, el cual, puede variar de pesca en pesca; en este caso, se considera una producción de 700 tinas, en donde, en la parte de corte solo el 55\% de la extracción de la pinchagua es válido para su producción, donde se lleva a las fábricas, pasa por un proceso de producción adecuado, para su posterior comercialización, como se visualiza en la Tabla 9.

Tabla 9

\section{Productividad en función del salario para eviscerado y recolectores}

\begin{tabular}{llll}
\hline Descripción & Eviseradores & $\begin{array}{l}\text { Recolectores } \\
\text { (Sardina) }\end{array}$ & $\begin{array}{l}\text { Recolectores } \\
\text { (Desperdicios) }\end{array}$ \\
\hline $\mathbf{N}^{\circ}$ de Trabajadores & 30 & 2 & 2 \\
Producción Mensual (Tinas) & 700 & 385 & 245 \\
\% de Corte & 55 & 55 & 35 \\
Total de Producción (después del eviscerado) & 385 & 385 & 245 \\
Valor & $\$ 4,50$ & $\$ 0,60$ & $\$ 0,80$ \\
Pago Total & $\$ 1732,50$ & $\$ 231,00$ & $\$ 196,00$ \\
Pago a C/Trabajador & $\$ 57,75$ & $\$ 115,50$ & $\$ 98,00$ \\
Total de productividad (por persona) & $\mathbf{1 2 , 1 2}$ & $\mathbf{3 , 3 3}$ & $\mathbf{2 , 5 0}$ \\
\hline
\end{tabular}

Fuente: Elaboración propia, 2020.

c. Productividad laboral: Unidades producidas / horas - hombres empleados.

De acuerdo a la aplicación de la fórmula, en este indicador se puede evidenciar que el nivel de productividad con respeto a las horas, se muestra que es considerablemente moderado, puesto que en el barco 15 personas llevan consigo 3,24 tinas por horas mensuales. Así mismo, el nivel de productividad respecto a las horas mensuales en la panga es de 3,24 tinas por horas mensuales, en los cargadores son 38,89 tinas por horas mensuales, el nivel de productividad respecto a las horas mensuales es de 21,39 tinas por horas mensuales; por otro lado, se encuentran las tinas de desperdicios, que tiene un total de 13,61 tinas por horas mensuales, cantidad que es similar al referirse a los choferes, aquella que se visualiza en la Tabla 10. 
Tabla 10

\section{Productividad laboral}

\begin{tabular}{|c|c|c|c|c|c|c|}
\hline \multirow{2}{*}{$\begin{array}{c}\mathrm{N}^{\circ} \mathrm{de} \\
\text { Trabajadores }\end{array}$} & \multirow{2}{*}{ Cargos } & \multicolumn{2}{|c|}{ Horas hombres } & \multirow{2}{*}{$\begin{array}{l}\text { Días al } \\
\text { mes }\end{array}$} & \multirow{2}{*}{$\begin{array}{c}\text { Producción } \\
\text { Mensual }\end{array}$} & \multirow{2}{*}{$\begin{array}{c}\text { Total de } \\
\text { Productividad }\end{array}$} \\
\hline & & $\begin{array}{l}\text { HORAS } \\
\text { MES }\end{array}$ & $\begin{array}{l}\text { HORAS } \\
\text { POR DÍA }\end{array}$ & & & \\
\hline 15 & Barco & 216 & 12 & 18 & 700 & 3.24 \\
\hline 6 & Pangas & 216 & 12 & 18 & 700 & 3.24 \\
\hline 5 & Cargadores & 18 & 1 & 18 & 700 & 38.89 \\
\hline 30 & Evisceradores & 18 & 1 & 18 & 700 & 38.89 \\
\hline \multicolumn{7}{|c|}{ Recogedores } \\
\hline 2 & $\begin{array}{l}\text { Recolectores } \\
\text { (Sardina) }\end{array}$ & 18 & 1 & 18 & 385 & 21,39 \\
\hline 2 & $\begin{array}{l}\text { Recolectores } \\
\text { (Desperdicios) }\end{array}$ & 18 & 1 & 18 & 245 & 13,61 \\
\hline \multicolumn{7}{|c|}{ Choferes } \\
\hline 2 & Chofer (Sardina) & 18 & 1 & 18 & 385 & 21,39 \\
\hline 2 & $\begin{array}{l}\text { Chofer } \\
\text { (Desperdicio) }\end{array}$ & 18 & 1 & 18 & 245 & 13,61 \\
\hline 64 & & & & & & \\
\hline
\end{tabular}

Fuente: Elaboración propia, 2020.

De acuerdo con Zambrano-Alcívar y Zambrano-Castro (2020), las empresas que participan en el eslabón de "pesca" cuentan con barcos pesqueros adaptados con cámara de refrigeración para movilizar el producto; algunas de estas empresas realizan procesos sobre la materia prima (atún) a fin de conservar el producto durante la jornada de pesca.

Por otra parte, Torres (2015) menciona que en el componente de comercialización en la estructura de la cadena de valor del sector de pesca en altamar, está relacionado con la acción de venta de los productos al consumidor final; sin embargo, para esta investigación, el componente principal de la cadena de valor es la parte de evisceración, el cual, aporta mayor valor a los pescadores del sector, discrepando un poco al autor, esto de acuerdo al sitio de la investigación, pueden variar los resultados.

Asimismo, Cedeño y Mendoza
(2013), argumenta que la pesca ha generado crecimiento económico en la ciudad de Crucita, puesto que la mayor parte de los habitantes se dedican a la captura de la pesca, y el resto a otras actividades. Coincidiendo con esta investigación, puesto que hasta la actualidad en esta ciudad la pesca es considerada la actividad principal para la generación de recursos económico como también el desarrollo de la ciudad.

De igual manera, Martínez (2012) plantea que el sistema de comercialización de la pinchagua curtida o sardina, es una actividad prometedora en este medio, fortaleciendo el ingreso económico de los pescadores artesanales, comerciantes mayoristas y minoristas, por la aceptación que tiene dentro del mercado nacional; no obstante, según el estudio realizado, no existe el apoyo suficiente a este sector, e incluso se presentan 
ciertas limitaciones al momento de extraer la pinchagua, desmotivando a los habitantes de este sector.

Por otra parte, Roldan y Avelovich (2020) plantean que los pescadores artesanales esperan una obra que restituya un lugar y un territorio donde desplegar con ventaja sus prácticas cotidianas. Quienes viven del río y lo conocen de una forma tan profunda como práctica, anhelan la formación de ese territorio de producción artesanal y colaboración de saberes.

Una vez recolectada la información, se establecen los elementos conceptuales que proporciona cada autor, los cuales, se direccionan en gran parte hacia la actividad industrial pesquera, agregar valor y hacer frente a la economía informal, como lo mencionan Martínez (2012); Cedeño y Mendoza (2013); y Torres (2015); no obstante, se concentran en otros aspectos que para los pescadores que viven el día a día su producción, lo descartan y consideran otros medios, como aprovechar los recursos al máximo, resaltando que el eslabón más importante de la cadena de valor de la pinchagua es el proceso eviscerado, eslabón que genera una mayor cantidad de empleo a los habitantes.

\section{Conclusiones}

Con la realización de este estudio se busca generar un mayor aporte de conocimiento acerca del procesamiento adecuado de la pinchagua, a través de la descripción detallada de todos los procesos presentes en los eslabones de este producto, a su vez, se identifica la cantidad de recursos que se utilizan en las empresas para generar un producto de calidad como es la pinchagua y la utilización de los desperdicios, de este modo se ve reflejada la eficiencia de los recursos generales y humanos, que han permitido el desarrollo de esta investigación .

Así también, entre las limitaciones de este estudio, se encuentra la facilidad de obtener información inmediata, debido a que el tiempo para los pescadores es muy valioso.
Respecto a las futuras líneas de investigación, está orientada a la gestión y dirección estratégica organizacional.

\section{Referencias bibliográficas}

Álvarez, R., Núñez, L., Calderón, F., y Mendoza, E. (2020). Producción y comercialización de productos de curtiembre de piel de pescado, Santa Elena - Ecuador. Revista de Ciencias Sociales (Ve), XXVI(4), 353367. $\underline{\text { https://doi.org/10.31876/rcs. }}$ v26i4.34667

Banco Central del Ecuador - BCE (2019). Cifras de las cuentas nacionales. BCE. https://www.bce.fin.ec/index. $\mathrm{php} /$ informacioneconomica/sectorreal _

Cedeño, C. J. y Mendoza, D. C. (2013). Análisis de la exportación de la pesca y su impacto en la generación de empleo en la ciudad de Crucita en el periodo 2008-2012 [Tesis de pregrado, Universidad Laica Eloy Alfaro de Manabí]. https://1library.co/document/ dy47rjry-analisis-exportacionesblanca-impacto-generacion-empleociudad-periodo.html

Donovan, C. (2006). Identificación de las oportunidades de mercado y mercadeo en Cadenas de Valor: Una guía para facilitadores del desarrollo empresarial rural. http://www.sidalc. net/repdoc/a2319e/a2319e.pdf

Fontalvo, T. J., De La Hoz, E. J., y Morelos, J. (2018). La productividad y sus factores: Incidencia en el mejoramiento organizacional. Dimensión Empresarial, 16(1), 47-60.

Galarza, E., y Kámiche, J. (2015). Pesca artesanal: Oportunidades para el desarrollo regional. Documento de Investigación, 3. Universidad del 
Zambrano Montesdeoca, Jasson Luis; Palacios Cedeño, Nohemi Monserrate; Ceme Macias, Cristina Auxiliadora y Intriago Zambrano, Mario José

Cadena de valor de la pinchagua y su productividad: Sitio Los Arenales

parroquia Crucita-Ecuador

Pacífico. https://repositorio.up.edu.pe/ bitstream/handle/11354/1013/DI3.pdf

García, A. (2011). Productividad y reducción de costos: Para la pequeña y mediana industria. Trillas.

González, N., Prado, M., Castro, R., Solano, F., Jurado, V., y Peña, M. (2017). Análisis de la pesquería de peces pelágicos pequeños en el Ecuador (1981-2007). Instituto Nacional de Pesca. https://institutopesca.gob. ec/wp-content/uploads/2017/07/ P e c e s - pe 1\%c 3\%a $1 \mathrm{~g}$ i c os peque $\%$ c $3 \%$ b1os-1981-2007.pdf

González N., y Solís E. (2010). Características biológico - pesqueras y proceso de elaboración de enlatados de la pinchagua (opisthonema spp) en Ecuador. Boletín Cientifico y Técnico, 20(7), 19-46.

Martínez, R. B. (2012). Sistema de comercialización de la pinchagua (opisthonema bulleri) encurtida y el fortalecimiento de la pesca artesanal en la zona sur de Manabí, periodo 2012 [Tesis de pregrado, Universidad Estatal del Sur de Manabí]. http://repositorio.unesum. edu.ec/handle/53000/432

Miranda, J., y Toirac, L. (2010). Indicadores de productividad para la industria dominicana. Ciencia y Sociedad, $X X X V(2) . \quad 235-290 . \quad$ https://doi. org/10.22206/CYS.2010.V35I2. PP235-290

Monroy, L. L., y Simbaqueaba, N. (2017). La importancia de los indicadores de gestión en las organizaciones colombianas [Tesis de pregrado, Universidad de La Salle]. https:// ciencia.lasalle.edu.co/administracion de empresas/1477

Organización de las Naciones Unidas para la Alimentación y la Agricultura - FAO (2015). Desarrollo de cadenas de valor alimentarias sostenibles: Principios rectores. FAO. https://www.fao.org/3/ i3953s/i3953s.pdf

Pazmiño-Morteira, A. I., y Vera-Santana, A. (2020). Eviscerado en la pesca artesanal, y su impacto ambiental en la caleta pesquera del recinto "Los Arenales", parroquia Crucita, segundo semestre del 2013. Revista Científica Arbitrada de Posgrado y Cooperación Internacional CLAUSTRO, 3(6), 2-11. https://publicacionescd.uleam.edu.ec/ index.php/claustro/article/view/133

Porter, M. (2006). Ventaja Competitiva: Creación y sostenimiento de un desempeño superior. Grupo Editorial Patria.

Rodríguez, C. (2017). Variación operacional de un vehículo autónomo submarino [Tesis de pregrado, Universidad Politécnica de Cartagena]. https:// repositorio.upct.es/xmlui/bitstream/ handle/10317/8262/pfc-rod-var. pdf? sequence $=1 \&$ is Allowed $=y$

Roldan, D., y Arelovich, L. (2020). Territorios en disputa. Los pescadores, la ribera, el urbanismo y el acuario del Paraná (Rosario, Argentina). UniversitasXXI. Revista de Ciencias Sociales y Humanas, (32), 77-98, https://doi. org/10.17163/uni.n32.2020.04

Torres, L. (2015). Diagnóstico de la cadena de valor de la pesca ecuatoriana de atún en altamar [Tesis de maestría, Universidad de Guayaquil]. http:// repositorio.ug.edu.ec/bitstream/ redug/12125/1/DIAGNOSTICO $\% 20$ DE\%20LA \%20CADENA\%20 PRODUCTIVA \%20 DE \%20 VALOR\%20DE\%20LA\%20 PESCA $\% 20$ ECUATORIANA $\% 20$ DE\%20AT\%C3\%9AN\%20EN\%20 ALTAMAR $\% 20 \mathrm{~A} \% \mathrm{C} 3 \% 910 \mathrm{O} \% 20$ 2012.pdf

Villegas, E., Alava, H. E., Ponce, J. E., y Palacios, D. L. (2020). Productividad 
total factorial y diferencias de ingreso a nivel internacional: 19502017. Revista de Ciencias Sociales (Ve), XXVI(3), 327-342. https://doi. org/10.31876/rcs.v26i3.33252
Zambrano-Alcívar, J. I., y Zambrano-Castro, L. C. (2020). Análisis de la industria atunera: Clúster, cadena de valor productiva y productividad. 593 Digital Publisher CEIT, 5(5-1), $267-$ 268. $\quad$ https://doi.org/10.33386/593 dp.2020.5-1.358 